\title{
HISTOMETRIA E FORMA TESTICULARES ASSOCIADAS À QUALIDADE SEMINAL EM MACHOS NELORE (Bos taurus indicus) CRIADOS EXTENSIVAMENTE NO SUDOESTE DE GOIÁS
}

\author{
Henrique Trevizoli Ferraz'1 Marco Antônio de Oliveira Viư1, Benedito Dias de \\ Oliveira Filho ${ }^{1}$, Dyomar Toledo Lopes ${ }^{1}$, Alessandra Feijó Marcondes Viu¹, Maria \\ Lúcia Gambarini ${ }^{1}$ \\ 1 UFG \\ Correspondência: Henrique Trevizoli Ferraz: htferraz@gmail.com
}

\begin{abstract}
RESUMO: Desenvolveu-se este estudo com o intuito de verificar as associações entre a forma dos testículos, as alterações histológicas no parênquima testicular e a qualidade seminal. Quarenta e dois animais, entre 30 e 36 meses de idade e que seriam castrados para terminação, tiveram o sêmen coletado. Amostras de três porções (proximal, intermediária e distal) de seus testículos foram colhidas e fixadas. Realizou-se a avaliação andrológica destes animais e a preparação de lâminas histológicas para avaliação do parênquima testicular. Foram avaliados: forma testicular; movimento massal (MMAS); vigor espermático (VIG); motilidade individual progressiva (MOT); concentração espermática (CONC); defeitos espermáticos maiores (DEFMAI), menores (DEFMEN) e totais (DEFTOT); distância média entre os túbulos seminíferos (DISTMED); espessura média do epitélio seminífero (ESPMED); e percentagem média de colágeno intersticial (COLMED). Animais com testículo classificado como longo apresentaram menor DISTMED, maior ESPMED e menor COLMED $(P<0,05)$, além de valores superiores quanto aos aspectos físicos e morfológicos do ejaculado. A DISTMED foi menor e a ESPMED foi maior na porção mais distal do testículo $(P<0,05)$. As correlações de DISTMED, ESPMED e COLMED com os DEFTOT foram, respectivamente, 0,51; 0,39 ; e $0,29(P<0,001)$. Assim, pode-se concluir que: testículos de forma alongada sofrem menos lesões teciduais no parênquima testicular, apresentando maior produção e melhor qualidade seminal; as porções distais dos testículos demonstram menos lesões ao tecido espermático; e as menores distâncias entre os túbulos seminíferos, a maior espessura do epitélio seminífero e a menor concentração de colágeno intersticial nos testículos estão relacionadas à melhoria da qualidade seminal.
\end{abstract}

Palavras-chave: andrologia; bovino; reprodução; sêmen; zebuíno

\section{TESTICULAR HISTOMETRY AND SHAPE ASSOCIATED WITH SEMINAL QUALITY IN NELLORE (Bos taurus indicus) MALES EXTENSIVELY RAISED IN SOUTHWEST OF GOIÁS}

\begin{abstract}
This study was developed to verify the associations between the shape of the testicles, the histological changes in testicular parenchyma and seminal quality. Forty two animals, between 30 and 36 months of age who were castrated for termination, had the semen collected. Samples of three portions (proximal, middle, and distal) of the testes were harvested and fixed. It was do breading soundness evaluation of these animals and preparation of slides for histological evaluation of testicular parenchyma. Were evaluated: testicular form; grass movement (MMAS); spermatic vigor (VIG); individual progressive motility (MOT); sperm concentration (CONC); major (DEFMAI), lower (DEFMEN) and total (DEFTOT) sperm defects; average distance between the seminiferous tubules (DISTMED); the average thickness of the seminiferous epithelium (ESPMED); and average percentage of interstitial collagen (COLMED). Animals with testicular classified as long had lower DISTMED, higher ESPMED, lower COLMED, and higher values regarding physical and morphological semen. The DISTMED was lower and ESPMED was higher in the distal portion of the testis. The correlations of DISTMED, ESPMED and COLMED with DEFTOT were respectively 0.51 ; 0.39; and 0.29. Thus, one can conclude that: elongated testes suffer less tissue damage in the testicular parenchyma, with higher production and better semen quality; the most distal portions of the testis show less tissue damage sperm; and the smallest distances between the seminiferous tubules, the greater thickness of the seminiferous epithelium and the lowest concentration of interstitial collagen in the testis are related to the improvement of sperm quality..
\end{abstract}

Key Words: andrology; bovine; reproduction; semen; zebuine 

taurus indicus) criados extensivamente no sudoeste de Goiás

\section{INTRODUÇÃO}

O parênquima testicular é composto por dois compartimentos principais: o compartimento tubular e 0 compartimento intersticial ou intertubular. O primeiro não é inervado nem vascularizado, constituindo a maior parte do testículo e sendo formado pela túnica própria, pelo epitélio seminífero e pelo lúmen. No compartimento intertubular estão localizadas as células de Leydig, vasos sanguíneos e linfáticos, nervos, fibroblastos e fibras de tecido conjuntivo, macrófagos, linfócitos e, ocasionalmente, mastócitos (FRANÇA e CHIARINI-GARCIA, 2005).

Para produção de espermatozoides férteis, a temperatura testicular dos touros deve ser de 4,0 a $5,0^{\circ} \mathrm{C}$ abaixo da corporal. Assim, animais submetidos à temperatura ambiente elevada podem apresentar elevação da temperatura corporal e, consequentemente, da testicular, afetando a qualidade do sêmen (KASTELIC et al., 1996). Vários trabalhos mostraram que 0 insulto térmico dos testículos resulta em diminuição da produção e qualidade espermática (BRITO et al., 2002; Fernandes et al., 2008). Adicionalmente, Fuerst-Waltl et al. (2006) observaram, em touros da raça Simental criados na Austrália, que a temperatura ambiental entre 5,0 e $15,0^{\circ} \mathrm{C}$ foi a que apresentou melhores resultados na produção seminal e Viu (2009) relatou tendência ao aumento das patologias espermáticas de touros Nelore andrologicamente avaliados em épocas de elevada temperatura ambiental.

Kastelic et al. (2001) encontraram correlação positiva entre a temperatura da superfície da bolsa escrotal e a temperatura testicular. Adicionalmente, Gábor et al. (1998) observaram correlação negativa entre a temperatura da superfície escrotal e a motilidade espermática. Segundo Kastelic et al. (1997), em temperatura ambiente de $25,0^{\circ} \mathrm{C}$, a diferença de temperatura da superfície escrotal entre o polo proximal e o distal do testículo atingiu valores de $1,5^{\circ} \mathrm{C}$, reforçando a hipótese de que, conforme penetra no parênquima testicular, o sangue arterial continua sendo refrigerado.

Alterações na forma do cone vascular testicular ou mesmo na forma dos testículos e bolsa escrotal podem afetar na forma de dissipação de calor e, consequentemente, na temperatura testicular (BAILEY et al., 1996; BRITO et al., 2002; BRITO et al., 2004). De acordo com Unanian et al. (2000), as formas testiculares mais alongadas apresentam vantagens morfofisiológicas, sendo apontadas como favoráveis à reprodução.

Dentre as principais causas de degeneração testicular nos animais criados em condições tropicais destacam-se as altas temperaturas ambientais (FUERST-WALTL et al., 2006), causando o aumento do metabolismo do tecido espermático e a consequente hipóxia do parênquima testicular, além da diminuição dos receptores de $\mathrm{FSH}$, levando à redução da produção e qualidade espermática (BRITO et al., 2002; KOIVISTO et al., 2008).

O quadro seminal em touros expostos ao insulto térmico começa a se modificar após 11 a 14 dias (Brito et al., 2003), com diminuição da concentração espermática, da motilidade individual progressiva, do vigor espermático e da porcentagem de espermatozoides morfologicamente normais (FERNANDES et al., 2008; KOIVISTO et al., 2008). Os efeitos deletérios da insulação dependem do tempo de agressão térmica (ARTEAGA et al., 2005) e podem ser reversíveis em função da severidade das alterações causadas no epitélio seminífero 
(NAGESWARA-RAO, 1997). Cerca de sessenta dias após a retirada da fonte de calor intenso o quadro seminal pode voltar ao normal, desde que a lesão tecidual não tenha sido extremamente grave (BRITO et al., 2002), num fenômeno conhecido como reversibilidade (ROBERTS, 1979).

De acordo com Buergelt (1997), histologicamente, a degeneração testicular é caracterizada pela circunferência irregular dos túbulos seminíferos, colapso tubular, estreitamento da lâmina basal dos túbulos e reação inflamatória intersticial, incluindo a deposição de colágeno na resolução do processo inflamatório (Jones et al., 2000). Hoflack et al. (2008), estudando touros da raça Belgian Blue, observaram aumento na percentagem de colágeno intersticial testicular e sua correlação $(P<0,05)$ com a percentagem de espermatozoides normais $(r=-0,47)$, defeitos primários ( $r$ $=0,48)$ e degeneração testicular $(r=$ $0,63)$ nestes animais.

A literatura consultada é pobre quanto a trabalhos relacionando alterações histológicas dos testículos, indicativas de degeneração testicular, associadas à diminuição da produção ou qualidade seminal em bovinos. Assim, considerando-se que os touros utilizados no Brasil são criados, predominantemente, de maneira extensiva e que, portanto, estão constantemente expostos aos efeitos das temperaturas ambientais elevadas, desenvolveu-se este estudo com o intuito de verificar as possíveis associações entre a forma dos testículos, as alterações histológicas no parênquima testicular e a qualidade seminal de touros Nelore criados extensivamente na região Centro-Oeste do Brasil, mais especificamente no Sudoeste de Goiás.

\section{MATERIAL E MÉTODOS}

Este estudo foi desenvolvido na Fazenda Santa Rosa, localizada no município de Caçu, sudoeste do Estado de Goiás, durante o mês de outubro de 2008. O conjunto de dados deste trabalho provém de 42 avaliações andrológicas realizadas em machos da raça Nelore de um rebanho comercial destinado ao corte, com idade variando entre 30 e 36 meses, criados extensivamente em pastagem de Brachiaria brizantha cv. Marandú. Foram estudadas as características reprodutivas: forma testicular; movimento massal (MMAS); vigor espermático (VIG); motilidade individual progressiva (MOT); concentração espermática (CONC); e defeitos espermáticos maiores (DEFMAI), menores (DEFMEN) e totais (DEFTOT).

Os touros foram submetidos a exame clínico geral e do sistema reprodutor de acordo com as recomendações do Colégio Brasileiro de Reprodução Animal (CBRA, 1998). As medidas de comprimento e largura dos testículos foram obtidas com o uso de paquímetro digital. O sêmen foi colhido pelo método de eletroejaculação e uma alíquota foi avaliada imediatamente para CONC (sptz/mL), MOT (0 a 100\%), VIG (0 a 5) e MMAS (0 a 5), todos em microscopia óptica com aumento de 100 vezes. Em seguida foi realizada a avaliação da morfologia espermática, usando microscopia de contraste de fase em aumento de 1.000 vezes, agrupando-se as patologias espermáticas em DEFMAI, DEFMEN e DEFTOT (CBRA, 1998). Com base nas informações obtidas determinaram-se as categorias por aptidão reprodutiva, sendo considerados aptos à reprodução aqueles com MOT $\geq 50 \%$, VIG $\geq 3$ e DEFTOT < 30\% e inaptos à reprodução aqueles com MOT $<50 \%$, VIG $<3$ ou DEFTOT > 30\%. 

taurus indicus) criados extensivamente no sudoeste de Goiás

Quanto à determinação da forma testicular, a classificação de cada animal foi realizada obtendo-se a razão entre a média das larguras do testículo esquerdo e direito e a média dos comprimentos dos dois testículos, de acordo com o método empregado por Bailey et al. (1996) e Bailey et al. (1998), obtendo-se as seguintes classes: testículos com formato longo (razão $\leq$ $0,5)$; longo-moderado $(0,51$ a 0,625$)$; longo-oval $(0,626$ a 0,750$)$; oval-esférico $(0,751$ a 0,875$)$; e esférico (razão > $0,875)$.

Para o estudo das características histológicas testiculares foram colhidas amostras de tecido dos dois testículos de cada animal após a castração. Para tanto, foram realizados três cortes transversais nas gônadas, sendo um no polo mais proximal, um na região intermediária e outro no pólo distal. Imediatamente após a colheita, este material foi devidamente armazenado e identificado em cassetes específicos para histologia, sendo então mantido em formol a $10 \%$. Na etapa laboratorial, após a fixação em formol as amostras foram submetidas à desidratação em doses crescentes de álcool $(70,80,90$ e $100{ }^{\circ} \mathrm{GL}$ ) e diafanizadas em xilol por 90 minutos. Posteriormente, foram incluídas em parafina histológica. A microtomia do material foi realizada em micrótomo automático, obtendo-se cortes de seis micras para confecção das lâminas histológicas.

As características histológicas avaliadas foram: a distância entre os túbulos seminíferos $(\mu \mathrm{m})$, a espessura do epitélio seminífero $(\mu \mathrm{m})$ e a percentagem de colágeno intersticial. Para determinação de quantas repetições destas medidas seriam necessárias para obtenção de uma média estatisticamente representativa, foi utilizada a metodologia descrita por Williams (1977), que mostrou serem necessárias, pelo menos, 56 repetições.
Assim, foi possível obter as seguintes variáveis: distância média entre os túbulos seminíferos (DISTMED); espessura média do epitélio seminífero (ESPMED); e percentagem média de colágeno intersticial (COLMED), todas obtidas a partir da média das 56 repetições de cada porção testicular em todos os animais.

Para aferição da distância entre os túbulos e da espessura do epitélio seminífero as lâminas histológicas foram coradas utilizando-se o tricrômio de Masson. Posteriormente estas foram fotografadas em microscópio óptico com sistema digital de captura de imagens, possibilitando assim a mensuração feita com auxílio do software livre ImageJ. As imagens foram obtidas em aumento de 250 vezes, procedendo-se a leitura em ziguezague da lâmina e fotografando-se campos que apresentavam cortes transversais dos túbulos seminíferos. Em cada imagem foram feitas oito mensurações da distância entre os túbulos e oito da espessura do epitélio seminífero. Assim, foram necessárias sete fotos de cada lâmina para se obter as 56 repetições necessárias.

Para determinação da percentagem de colágeno intersticial, as lâminas foram coradas com o Picrosirius Red e fotografadas utilizando-se microscopia com luz polarizada. A mensuração da percentagem de colágeno também foi feita utilizando-se - software livre ImageJ, através da determinação da proporção de colágeno existente no campo fotografado. Este foi obtido em aumento de 32 vezes, após leitura em ziguezague das lâminas. Optou-se pelo menor aumento possível para se ter maior representatividade da região intersticial no campo analisado. Para se ter as 56 repetições necessárias, foram feitas 56 fotos digitais de cada porção dos testículos.

$O$ delineamento experimental foi o inteiramente casualizado, sendo os 
parâmetros obtidos analisados utilizando-se o pacote computacional SAS - v. 8.0 (SAS, 2000). Foram realizadas análises de crítica e consistência (frequências, distribuição de frequências e homogeneidade de variâncias). Mediante a utilização do programa computacional SAEG (2005) empregaram-se os seguintes testes: Lilliefors, para determinar se os erros das variáveis possuíam distribuição normal de probabilidade; e o de Bartlett, para determinar se as variáveis possuíam homogeneidade de variância dos erros experimentais. As características seminais e histológicas foram submetidas às análises de variância utilizando-se o procedimento General Linear Models (GLM) do programa computacional SAS - v. 8.0 (SAS, 2000). O teste de média " $\mathrm{t}$ " foi feito por meio da opção LSMEANS do procedimento GLM do programa computacional referido anteriormente. Para o estudo da intensidade com que se manifesta uma associação entre duas variáveis, optou-se pelo uso do coeficiente de correlação de Spearman, calculado pelo procedimento CORR do programa SAS - v. 8.0 (SAS, 2000).

\section{RESULTADOS E DISCUSSÃO}

A Tabela 1 mostra as médias, ajustadas pelos quadrados mínimos, das variáveis histológicas testiculares e de qualidade seminal (aspectos físicos e morfológicos) do presente estudo quando se considera a aptidão reprodutiva dos animais.

Os animais classificados como aptos à reprodução apresentaram menor DISTMED $(P<0,05)$, maior ESPEMED $(P<0,05)$ e menor COLMED $(P<0,05)$ do que aqueles classificados como inaptos (Tabela 1). Analisando-se estes achados pode-se dizer que nos animais aptos a espermatogênese é mais ativa, comprovada pela maior espessura do epitélio seminífero, uma vez que, de acordo com Banks (1991), a presença e os constituintes deste epitélio dependem da atividade espermatogênica dos túbulos. Provavelmente, o interstício dos testículos destes animais contém menos infiltrado inflamatório (FRANÇA e CHIARINI-GARCIA, 2005), comprovado pela menor DISTMED, além de uma proporção menor de colágeno, sugerindo que nesses animais as reações teciduais a agentes agressores, principalmente às elevadas temperaturas ambientais (Fuerst-Waltl et al., 2006), foi menor.

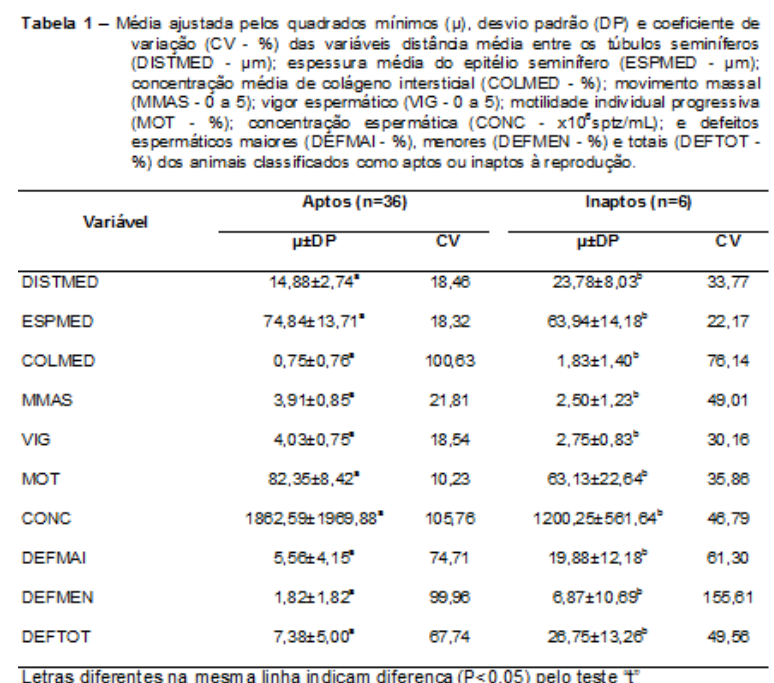

Todas as variáveis de qualidade seminal (MMAS, VIG, MOT, CONC, DEFMAI, DEFMEN e DEFTOT) mostraram-se favoráveis à reprodução $(\mathrm{P}<0,05)$ nos animais aptos (Tabela 1$)$. Isto é facilmente explicado considerando-se o fato de que estas mesmas variáveis são utilizadas como critério para classificação dos touros quanto à sua capacidade reprodutiva (CBRA, 1998).

$\mathrm{Na}$ Tabela 2 estão demonstradas as médias, ajustadas pelos quadrados mínimos, das variáveis histológicas e seminais dos animais classificados quanto à forma de seus testículos. Pode-se observar que aqueles com testículo classificado como longo 

taurus indicus) criados extensivamente no sudoeste de Goiás

apresentaram menor DISTMED $(P<0,05)$, maior ESPMED $(P<0,05)$ e menor COLMED $(P<0,05)$, além de valores desejáveis quanto aos aspectos físicos e morfológicos do ejaculado $(P<0,05)$, exceto na variável DEFMEN. Vale ressaltar que esta é uma variável muito sujeita a efeitos não pertinentes a alterações morfológicas do espermatozoide por falhas na espermatogênese, como, por exemplo, a contaminação da coleta com urina, que provoca o aumento destes defeitos (CBRA, 1998).

Os achados mostrados na Tabela 2 vão ao encontro do sugerido por Bailey et al. (1996) e Bailey et al. (1998), que propuseram a consideração do volume e da forma testiculares como preditores do potencial espermatogênico, além do consagrado perímetro escrotal, uma vez que, segundo esses mesmos autores, os testículos mais alongados teriam maior facilidade em dissipar calor do parênquima testicular para o ambiente. Unanian et al. (2000), avaliando animais da raça Nelore, também relataram melhor qualidade seminal em animais com testículos mais longos.

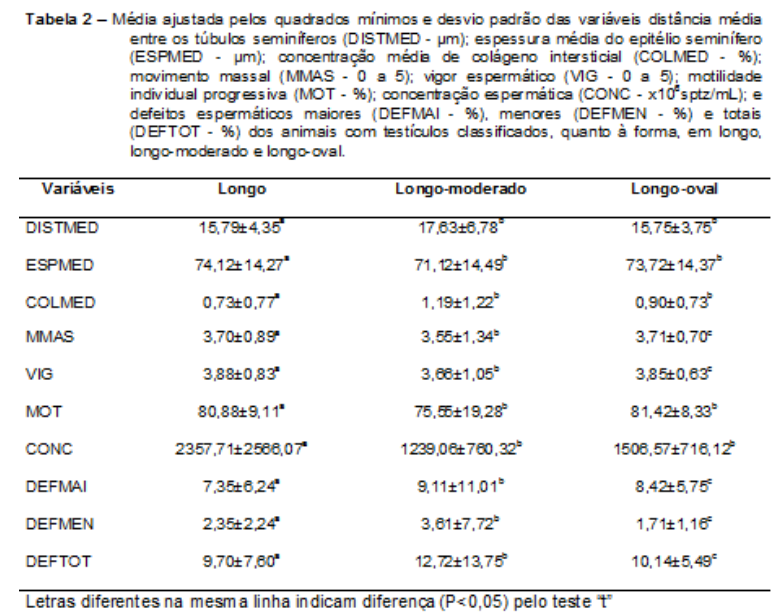

Analisando-se a Tabela 3 é possível verificar as variáveis histológicas em estudo nas diferentes porções do testículo (proximal, intermediária e distal). Conforme demonstrado nesta tabela a DISTMED foi menor $(P<0,05)$ e a ESPMED foi maior $(P<0,05)$ na porção mais distal do testículo, evidenciando menor agressão ao tecido espermático (França e Chiarini-Garcia, 2005) e maior atividade espermatogênica nesta região (Banks, 1991). Isto pode ser explicado pelo fato de que 0 sangue que chega aos testículos continua sendo refrigerado ao passar pelo parênquima, de tal maneira que atinge a porção distal com até $1,5^{\circ} \mathrm{C}$ a menos (Kastelic et al., 1997), tornando esta região menos sujeita ao calor conduzido pelo sangue da artéria testicular, uma vez que para a boa espermatogênese a temperatura testicular deve ser 4,0 a $5,0^{\circ} \mathrm{C}$ mais baixa que a temperatura corpórea (Kastelic et al., 1996). A maior COLMED $(P<0,05)$ na porção distal pode ser biologicamente explicada pelo fato de que este é oriundo da resolução do processo inflamatório (Jones et al., 2000) e o infiltrado inflamatório, por ser um fluido, tende a se acumular nas porções mais baixas do corpo. Adicionalmente, nas extremidades do corpo o retorno venoso é deficiente em função da menor pressão com que o sangue chega a essas regiões (Reece, 2006), diminuindo a drenagem do infiltrado inflamatório.

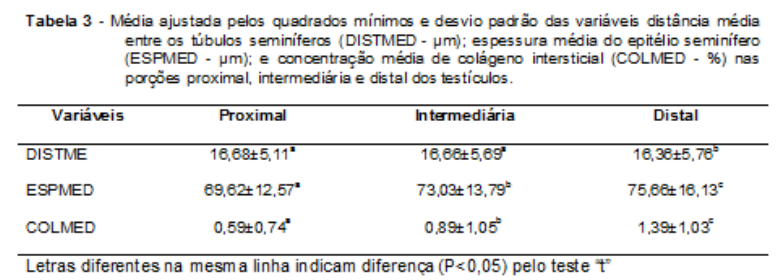

A Tabela 4 mostra as correlações fenotípicas entre as variáveis histológicas (DISTMED, ESPMED e COLMED) e aquelas relacionadas à morfologia espermática (DEFMAI, DEFMEN e DEFTOT). A DISTMED apresentou correlação de moderada a alta intensidade com os defeitos espermáticos $\quad(P<0,001)$, permitindo afirmar que quanto maior for 0 distanciamento entre os túbulos 
seminíferos, maiores serão as patologias seminais. A ESPMED demonstrou correlações negativas de moderada a alta intensidade com os defeitos espermáticos $\quad(P<0,001)$, mostrando que com a menor atividade espermatogênica e diminuição da espessura do epitélio seminífero (Banks, 1991) aumentam as alterações morfológicas seminais. A COLMED apresentou correlação de moderada intensidade com as patologias espermáticas $(P<0,001)$. Corroborando com estes achados, Hoflack et al. (2008) também observaram correlações entre 0 colágeno intersticial e anormalidades espermáticas/ degeneração testicular.

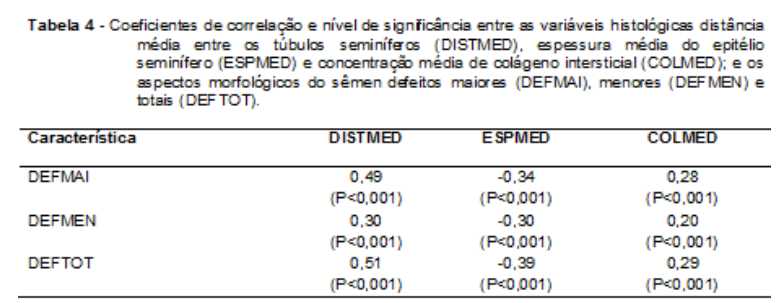

\section{CONCLUSÃO}

Com base nos resultados obtidos neste estudo pode-se concluir que: bovinos com testículos de forma alongada sofrem menos lesões teciduais no parênquima testicular, apresentando maior produção e melhor qualidade seminal; as porções distais dos testículos demonstram menos lesões ao tecido espermático, provavelmente por uma melhor termorregulação; e as menores distâncias entre os túbulos seminíferos, a maior espessura do epitélio seminífero e a menor concentração de colágeno intersticial nos testículos estão relacionados à melhoria da qualidade seminal.

\section{REFERÊNCIAS}

ARTEAGA, A. A.; BARTH, A. D.; BRITO, L. F. C. et al. Relationship between semen quality and pixel-intensity of testicular ultrasonograms after scrotal insulation in beef bulls. Theriogenology, v.64, p.408-415, 2005.

BAILEY, T. L.; MONKE, D.; HUDSON, R. S.; et al. Testicular shape and its relationship to sperm production in mature Holstein bulls.

Theriogenology, v.46, p.881-887, 1996.

BAILEY, T. L.; HUDSON, R. S.; POWE, T. A.; et al. Caliper and ultrasonographic measurements of bovine testicle and mathematical formula for determining testicular volume and weight in vivo. Theriogenology, v.49, p.581-598, 1998.

BANKS, W. J. Histologia veterinária aplicada, São Paulo: Manole, 1991. 629 p.

BRITO, L. F. C.; SILVA, A. E. D. F.; RODRIGUES, L. H.; et al. Effect of age and genetic group on characteristics of the scrotum, testes and testicular vascular cones, and on sperm production and semen quality in Al bulls in Brazil. Theriogenology, v.58, p.1175-1186, 2002.

BRITO, L. F. C.; SILVA, A. E. D. F.; BARBOSA, R. T.; et al. Effects of scrotal insulation on sperm production, semen quality, and testicular echotexture in Bos indicus and Bos indicus $\mathrm{X}$ Bos taurus bulls. Animal Reproduction Science, v.79, p.1-15, 2003.

BRITO, L. F. C.; SILVA, A. E. D. F.; UNANIAN, M. M.; et al. Sexual development in early and late-maturing Bos indicus and Bos indicus $x$ Bos taurus crossbred bulls in Brazil.Theriogenology, v.62, p.1198-1217, 2004.

BUERGELT, C. D. Color atlas of reproductive pathology of domestic animals. Saint Louis: Mosby, 1997. 219 p.

CBRA. Manual para exame andrológico e avaliação de sêmen animal/Colégio Brasileiro de Reprodução Animal. Belo Horizonte: CBRA, 1998. $49 \mathrm{p}$.

FERNANDES, C. E.; DODE, M. A. N.; PEREIRA, D.; SILVA, A. E. D. F. Effects of scrotal insulation in Nellore bulls on seminal quality and its relationship with in vitro fertilizing ability. Theriogenology, v.70, p.1560-1568, 2008. 

taurus indicus) criados extensivamente no sudoeste de Goiás

FRANÇA, L. R.; CHIARINI-GARCIA, H. Célula de Sertoli. In: CARVALHO, H. F.; COLLARESBUZATO, C. B. Células - uma abordagem multidisciplinar. Barueri: Manole, 2005. cap. 24, p.302-324.

FUERST-WALTL, B.; SCHWARZENBACHER, H.; PERNER, C.; SOLKNER, J. Effects of age and environmental factors on semen production and semen quality of Austrian Simmental bulls. Animal Reprod. Science, v.95, p.27-37, 2006.

GÁBOR, G.; SASSER, R. G.; KASTELIC, J. P.; et al. Morphologic, endocrine and thermographic measurements of testicles in comparison with semen characteristics in mature HolsteinFriesian breeding bulls. Animal Reproduction Science, v.51, p.215-224, 1998.

HOFLACK, G.; VAN DEN BROECK, W.; MAES, $D$.; et al. Testicular dysfunction is responsible for low sperm quality in Belgian Blue bulls.

Theriogenology, v.69, p.323-332, 2008.

JONES, T. C.; HUNT, R. D.; KING, N. W.

Patologia veterinária. 6.ed. São Paulo: Manole, 2000, 1415p.

KASTELIC, J. P.; COOK, R. B.; COULTER, G. H.; SAACKE, R. G. Insulating the scrotal neck affects semen quality and scrotal/testicular temperatures in the bull. Theriogenology, v.45, p.935-942, 1996.

KASTELIC, J. P.; COOK, R. B.; COULTER, G. $\mathrm{H}$.; et al. Contribution of the scrotum, testes, and testicular artery to scrotal/testicular thermoregulation in bulls at two ambient temperatures. Animal Reproduction Science, v.45, p.255-261, 1997.

KASTELIC, F.P.; COOK, R.B.; PIERSON, R.A.; COULTER, G.H. Relationships among scrotal and testicular characteristics, sperm production, and seminal quality in 129 beef bulls. The Canadian Journal of Veterinary Research, v.65, p.111-115, 2001.

KOIVISTO, M. B.; COSTA, M. T. A.; PERRI, S. H. V.; VICENTE, W. R. R. The effect the effect of season on semen characteristics and freezability in Bos indicus and Bos taurus bulls in the southeastern region of Brazil. Reproduction in Domestic Animals, v.42, p.715-726, 2008.

NAGESWARA-RAO, V. D. Influence of heat induced testicular degeneration on semen characteristics and testicular histology in rams.
Indian Veterinary Journal, v.54, p.719-726, 1997.

SAEG - Sistema para análises estatísticas, versão 9.0. Viçosa: FUNARBE, 2005.

SAS - User's Guide: statistics. 5. ed., Cary: SAS Institute, 2000. 1028 p.

REECE, W. O. Dukes - Fisiologia dos animais domésticos, 10.ed., Rio de Janeiro: Guanabara Koogan, 2006.

ROBERTS, S. J. Obstetricia veterinaria y patología de la reproducción (teriogenologia). Editora Hemisferio Sur S. A., 1979. $1021 \mathrm{p}$.

UNANIAN, M. M.; SILVA, A. E. D. F.; McMANUS, C.; CARDOSO, E. P. Características biométricas testiculares para avaliação de touros zebuínos da raça Nelore. Revista Brasileira de Zootecnia, v.29, p.136144, 2000.

WILLIAMS, M. A. Quantitative methods in Biology. In: GLAUBERT, A. M. Practical methods in electron microscopy. Amsterdam: Elsevier North-Holland Biomedical Press, 1977, $233 \mathrm{p}$.

VIU, M. A. O. Estudo Genético quantitativo e ambiental do potencial reprodutivo de touros Nelore criados no Centro-Oeste do Brasil. 2009. Goiânia, 118f. Tese (Doutorado em Ciência Animal) - Curso de Pós-Graduação em Ciência Animal, Universidade Federal de Goiás. 